\title{
UTILITY OF A SHORT PSYCHO-BEHAVIORAL ASSESSMENT FOR THE IDENTIFICATION OF OVERWEIGHT ADOLESCENTS AT RISK OF UNSUCCESSFUL WEIGHT LOSS.
}

\author{
Videira-Silva A., MSc 1,2, Fonseca H., MD, PhD, MPH ${ }^{2}$
}

1Pediatric University Clinic, Faculty of Medicine, University of Lisbon, Lisbon, Portugal. antonioascenso@campus.ul.pt 2 Pediatric Obesity Clinic, Hospital de Santa Maria, Lisbon, Portugal.

\section{Introduction \& Aim}

Psycho-behavioral characteristics should be assessed in order to personalize weight management programs. The most commonly used psychometric instruments take too long, making them unsuitable for the clinical office routine.

This study aimed to analyze whether a brief psycho-behavioral assessment can predict body mass index (BMI) z-score variations among overweight adolescents followed at a Pediatric Obesity Clinic.

\section{Methods}

A brief psycho-behavioral assessment including three questions regarding quality of motivation, self-efficacy and perception of importance to lose weight, was conducted.

Psycho-behavioral, pubertal and anthropometric data from 150 overweight adolescents (BMI $\geq \mathrm{p} 85$ ), aged 10-17, were retrospectively analyzed. Kruskal-Wallis non-parametric test, partial correlations controlling for sex, age and Tanner stage, and regression analysis were conducted.

\section{Methods - Study Design}

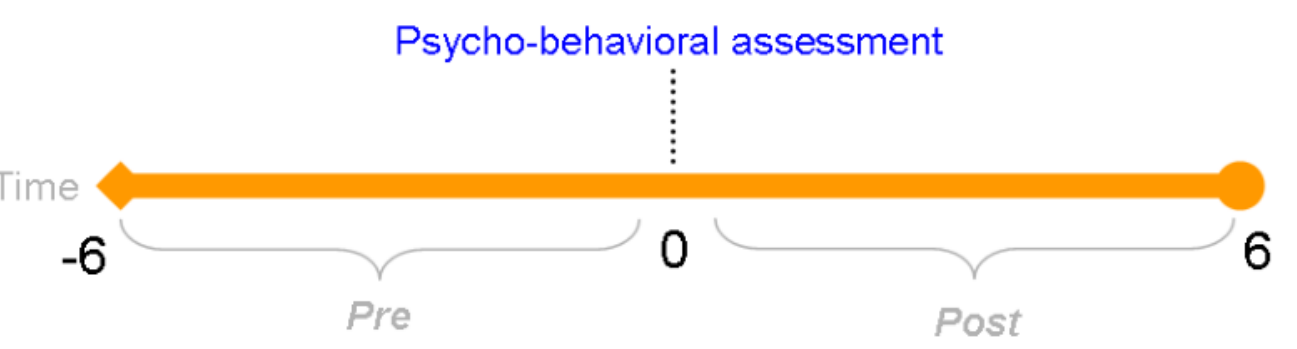

Keywords: Adolescents; Overweight; Weight loss; Motivation; Perception of importance to lose weight; Self-efficacy.

Figure 1. Association between quality of motivation and perception of importance to lose weight.

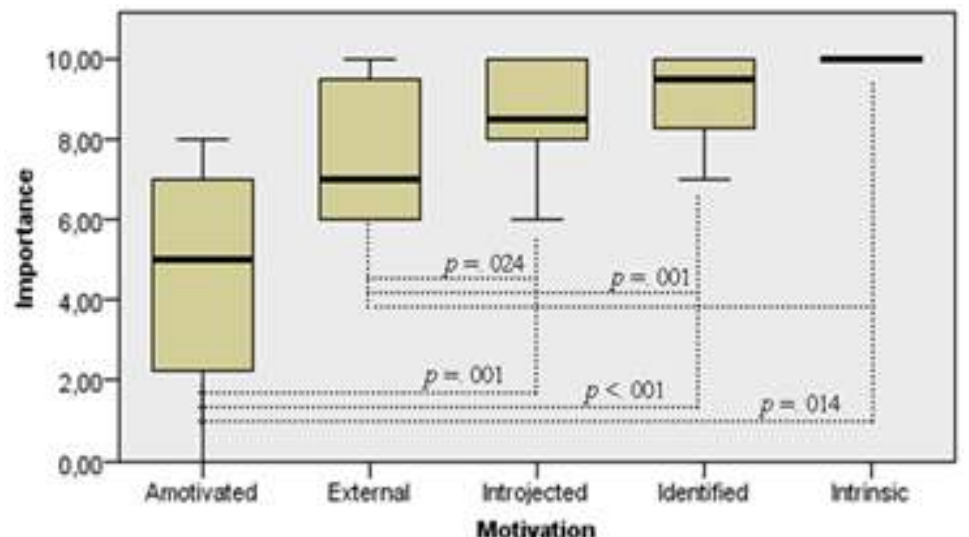

Figure 2. Association between quality of motivation and self-efficacy perception.

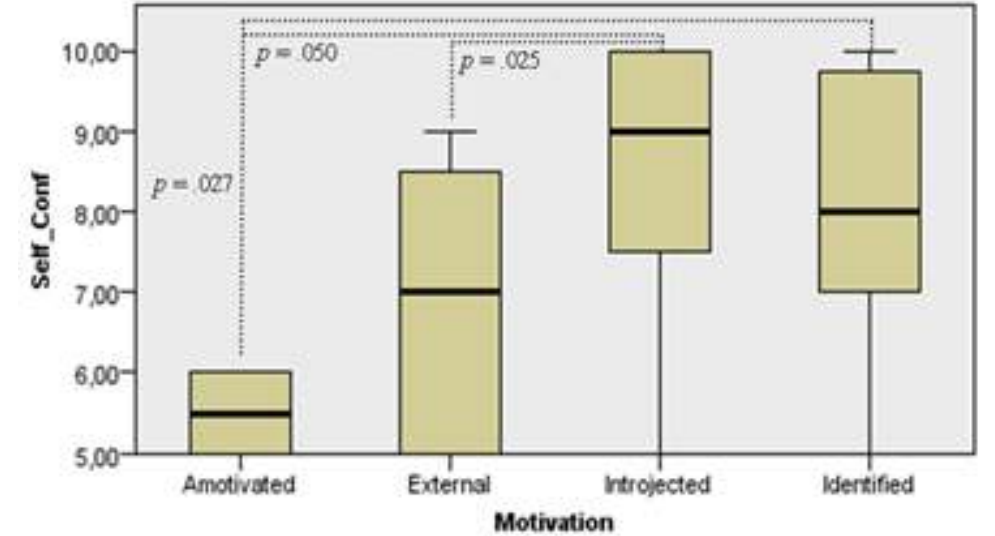

Legend: BMI- Body mass index BFM- Body fat mass; CRP- C-Reactive protein HDL-C- High-density lipoprotein cholesterol; HOMA-IR- Insulin resistance

Figure 4. Inter-relationship between motivation type, self-efficacy and perception of importance to lose weight, and BMI z-score variations.

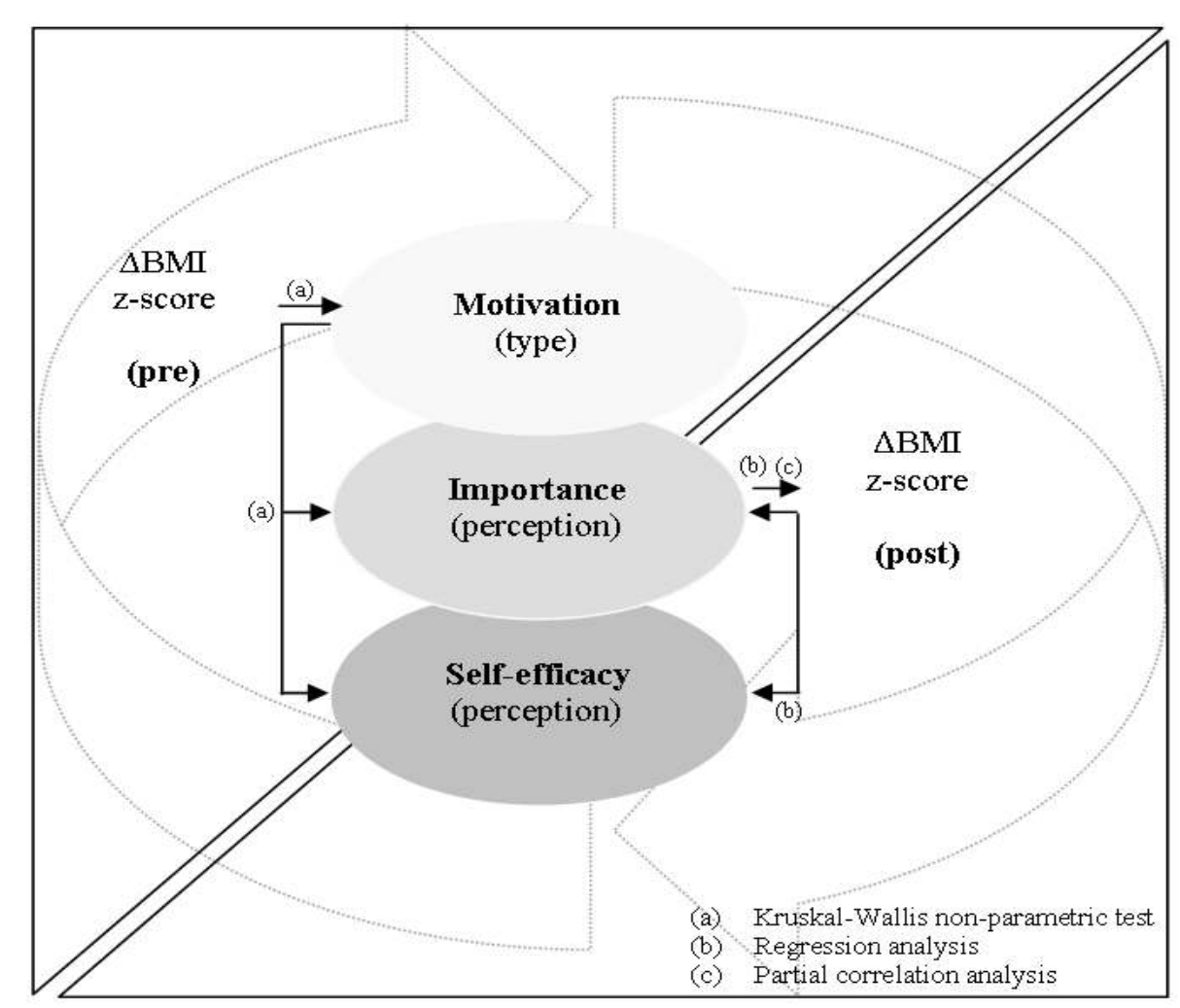

Figure 3. Association between BMI z-score variations pre-behavioral regulation assessment and quality of motivation.

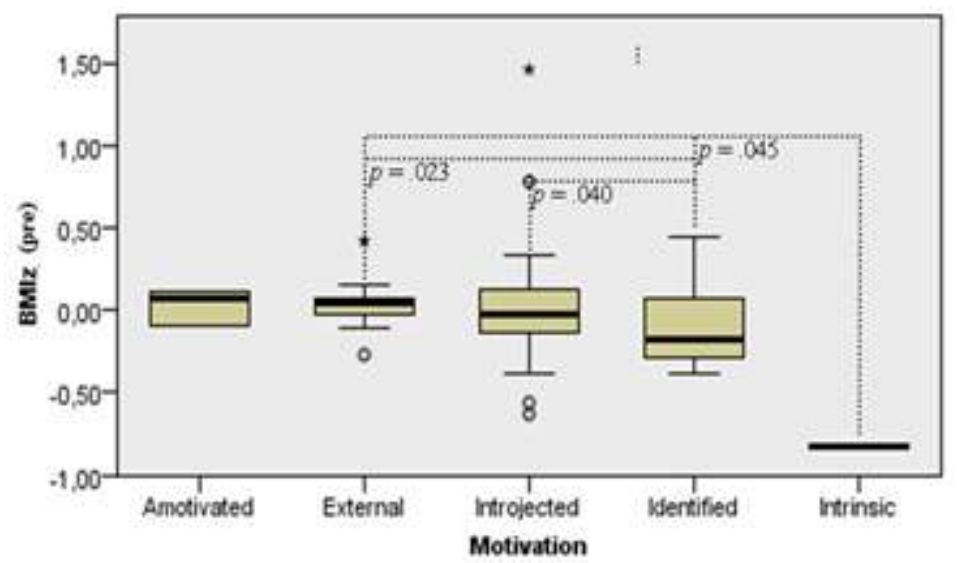
at risk of unsuccessful weight loss. draw more robust conclusions.

This study suggests that a brief psycho-behavioral assessment may predict BMI z-score variations, helping Health Professionals to identify overweight adolescents

Future studies including larger samples may enable to 